\title{
Investigation on Knowledge Awareness, Self-Protective Behaviors and SAS Anxiety of Nursing Students during the Epidemic of COVID-19
}

\author{
Huan-ze $\mathrm{Li}^{1}$, Hong-fang Chen ${ }^{1,2, *}$, Zheng-zheng Yao ${ }^{2}$, Miao-qing Zhuang ${ }^{1}$ \\ ${ }^{1}$ Shaanxi University of Traditional Chinese Medicine, Xianyang, Shaanxi, China. \\ ${ }^{2}$ Shaanxi Provincial Hospital of Traditional Chinese Medicine Nursing Department, Xi'an, Shaanxi, China.
}

How to cite this paper: Huan-ze $\mathrm{Li}$, Hong-fang Chen, Zheng-zheng Yao, Miao-qing Zhuang. (2020) Investigation on Knowledge Awareness, Self-Protective Behaviors and SAS Anxiety of Nursing Students during the Epidemic of COVID-19. International Journal of Clinical and Experimental Medicine Research, 4(4), 194-200. DOI: 10.26855/ijcemr.2020.10.013

Received: July 28, 2020

Accepted: August 26, 2020

Published: September 15, 2020

*Corresponding author: Hong-fang Chen, Shaanxi University of Traditional Chinese Medicine, Xianyang, Shaanxi, China; Shaanxi Provincial Hospital of Traditional Chinese Medicine Nursing Department, Xi'an, Shaanxi, China. Email: 13892881695@163.com

\begin{abstract}
Objective: To investigate the knowledge awareness, self-protective behaviors of nursing students and their effects on SAS anxiety during the epidemic of COVID-19. Methods: Convenient sampling method was used to anonymously survey nursing students based on the questionnaire star network. The self-prepared general information questionnaire, knowledge of new coronavirus knowledge, self-protective behaviors and SAS anxiety scale were used for investigation. Results: A total of 362 nursing students were surveyed. The anxiety state detection rate was $14.09 \%$. The SAS anxiety score was (33.64 \pm 6.17), which was higher than the norm of anxiety symptoms in Chinese normal people (29.78 $\pm 10.07, \mathrm{n}=1158)$. It is statistically significant $(\mathrm{P}<0.01)$. Knowledge awareness was positively correlated with self-preventive behaviors ( $\mathrm{r}=0.126, \mathrm{P}<0.05)$, knowledge awareness was negatively correlated with SAS anxiety $(r=-0.196, \mathrm{P}<0.01)$. Conclusion: The anxiety of nursing students during the epidemic of COVID-19 is prominent, which has a close interactive relationship with the knowledge and self-protective behaviors. It is necessary to adopt a combination of online and offline epidemic knowledge popularization, supervise the implementation of self-protective behaviors of nursing students, timely conduct psychological counseling, strengthen the professional guidance of nursing students, and alleviate the negative emotions of nursing students.
\end{abstract}

\section{Keywords}

COVID-19, 2019-nCoV, Nursing Students, Self-Protective Behavior, Anxiety

\section{Introduction}

The Corona Virus Disease 2019 (COVID-19) is a new-onset acute respiratory disease that is more infectious than SARS in 2003, spreads faster, and spreads more insidiously. On January 20, 2020, the National Health Commission of China included the disease as a Class B infectious disease stipulated in the Law of the People's Republic of China on the Prevention and Control of Infectious Diseases and adopted prevention and control measures for Class A infectious diseases [1]. All parts of the country have taken measures such as closed isolation, postponement of resumption of work after the Spring Festival, and postponement of school opening. On March 11, 2020, the World 
Health Organization (WHO) announced that COVID-19 has entered a global pandemic [2]. The global spread of new crown pneumonia not only brings immeasurable damage and threat to the physical and mental health of the people, but also severely impacts the global health and medical system. In the domestic fight against the epidemic, as of February 29, 2020, the National Health Commission of China has sent more than 42,000 medical personnel to Wuhan, Hubei [3], including 28,600 nurses, accounting for 68\% of the total number of medical teams. Medical staff not only face high-intensity work, but also face psychological pressure such as the risk of infection. According to statistics, as of February 29, 2020, more than 3,000 medical staff in China were infected with new coronary pneumonia [4]. Although the epidemic in China is basically under control, the global epidemic is still severe. There are reports in the United Kingdom that due to a severe shortage of medical staff, some nursing interns and retired medical staff have entered clinical first-line anti-epidemic positions [5]. Now, the world needs the knowledge and ability of nursing workers more than ever. Nursing students are the backup force of the nursing team, but the professional values of this group are not yet mature, and the spread of a large number of false news during the epidemic has caused various psychological stress phenomena such as fear and anxiety among nursing students. This study investigated the relevant knowledge, self-protection behavior and psychological anxiety state of nursing students in the face of the major epidemic of COVID-19, in order to provide a reliable basis for the intervention and adjustment of relevant education departments. The report is as follows.

\section{Objects and methods}

\subsection{Survey objects}

Using convenience sampling, students from five nursing colleges in China were selected, and nursing students were invited to fill out the questionnaire online based on the questionnaire star platform. A total of 362 nursing college students, nursing undergraduates, and nursing graduate students are included. The survey time is March 2020.

\subsection{Survey tools}

\subsubsection{General information}

Self-made general information questionnaire, including gender, educational level, whether to experience clinical internship, place of residence, the epidemic situation in the place of residence, the duration of daily attention to the epidemic, and the frequency of training related to the COVID-19in schools.

\subsubsection{New Coronary Pneumonia Related Knowledge Questionnaire}

According to the Guidelines for Public Protection of Pneumonia from COVID-19Infection [6] that Organized and compiled by the Bureau of Disease Control and Prevention of the National Health Commission of China, edited by the Chinese Center for Disease Control and Prevention. Investigate the knowledge of nursing students about COVID-19 during the epidemic. The options are all single-choice questions, a total of 15 questions, 4 points for the right choice, 0 points for the wrong choice, and a total score of 0-60 points. The higher the score, the better the knowledge about COVID-19.

\subsubsection{Questionnaire on self-protection behavior against COVID-19}

According to the Guidelines for the Public Protection of Pneumonia from COVID-19 [6], the questionnaire was compiled by itself to investigate the prevention and control actions taken by individuals during the epidemic. The options are all single-choice questions, a total of 10 questions, 4 points for the right choice, 0 points for the wrong choice, the total score is 0-40 points, the higher the score, the higher the compliance of self-protection behavior.

\subsubsection{Selfrating anxiety scale (SAS)}

Adopted by W. K. Zung [7] in 1971, a total of 20 items, using a 4-level score, used to assess the subjective feelings of the subjects in the last week, the higher the score, the more obvious the anxiety tendency. According to the Chinese norm (29.78 \pm 10.07$)$ result screening standard [8], according to the SAS standard score $\geq 50$, it is classified as positive. The reliability and validity of the scale are good, and it can better reflect the mental health of the population.

\subsection{Quality control}

The application questionnaire star platform invites students to fill out the questionnaire online. Set all items as "required answer", the same IP address can only answer once, and the answering time is less than 100 seconds as an invalid questionnaire. A total of 365 questionnaires were collected, and 3 invalid questionnaires were eliminated. There were 362 valid questionnaires, with an effective rate of $99.2 \%$. 


\subsection{Statistical processing}

The questionnaire star was used to download the original data to the SPSS database, and the SPSS25.0 software was used for data analysis. General information is described by frequency and composition ratio; the overall scores of knowledge awareness, self-prevention behavior, and SAS anxiety level are described by mean \pm standard deviation; Pearson correlation analysis method is used to analyze the correlation between knowledge awareness, self-preventive behaviors, and SAS anxiety level; Inspection level $\alpha=0.05$.

\section{Results}

\subsection{Investigate the basic situation of nursing students (Table 1)}

Table 1. Basic situation of nursing students $(n=362)$

\begin{tabular}{|c|c|c|}
\hline Factor & Number of cases & Percentage (\%) \\
\hline \multicolumn{3}{|l|}{ gender } \\
\hline male & 46 & 12.7 \\
\hline Female & 316 & 87.3 \\
\hline \multicolumn{3}{|l|}{ Education } \\
\hline Junior college & 97 & 26.8 \\
\hline Undergraduate & 253 & 69.9 \\
\hline Postgraduate & 12 & 3.3 \\
\hline \multicolumn{3}{|l|}{ Clinical Practice } \\
\hline Have experienced & 139 & 38.4 \\
\hline Not experienced & 223 & 61.6 \\
\hline \multicolumn{3}{|l|}{ Place of residence } \\
\hline Villages and towns & 205 & 56.6 \\
\hline county seat & 84 & 23.2 \\
\hline County-level city & 51 & 14.1 \\
\hline Capital city & 22 & 6.1 \\
\hline \multicolumn{3}{|l|}{ Adapt to the epidemic } \\
\hline Yes & 312 & 86.2 \\
\hline no & 50 & 13.8 \\
\hline \multicolumn{3}{|l|}{ Keep an eye on the epidemic } \\
\hline$<30$ minutes & 129 & 35.6 \\
\hline 30-60 minutes & 191 & 52.8 \\
\hline $60-120$ minutes & 25 & 6.9 \\
\hline$>120$ minutes & 17 & 4.7 \\
\hline \multicolumn{3}{|l|}{ Schoolprotection education } \\
\hline hardly & 7 & 1.9 \\
\hline less & 73 & 20.2 \\
\hline frequently & 282 & 77.9 \\
\hline \multicolumn{3}{|c|}{ Epidemic situation in place of residence } \\
\hline This community has & 16 & 4.4 \\
\hline This (city) county has & 192 & 53.0 \\
\hline This province has & 154 & 42.5 \\
\hline
\end{tabular}


3.2 The overall scores of nursing students' knowledge, self-protective behaviors, and SAS anxiety level survey under the COVID-19 (See Table 2)

Table 2. Nursing students' knowledge, self-protection behavior, SAS anxiety level score table under the COVID-19 (n = 362)

\begin{tabular}{cccc}
\hline Project & Scale full score (points) & Total average score $\left(\mathrm{X}^{2}\right.$, points) & Standardized scoring rate $(\%)$ \\
\hline Knowledge level & 60 & $38.41 \pm 7.31$ & 64.02 \\
Self-defense behavior & 40 & $34.92 \pm 4.03$ & 87.30 \\
SAS anxiety level & 80 & $33.64 \pm 6.17$ & 43.05 \\
\hline
\end{tabular}

Note: Standardized scoring rate $=$ total average score/scale full score $\times 100 \%$.

\subsection{Detection rate of SAS anxiety in nursing students under the COVID-19}

Among the 362 survey subjects, the detection rate of anxiety state was $14.09 \%$. The total average SAS score is (33.64 \pm 6.17 ), and the standardized score rate is 51 in which the standard score is $\geq 50$, which is higher than the normal anxiety symptoms of normal people in China $(29.78 \pm 10.07, \mathrm{n}=1,158)$. The difference is significant $(\mathrm{t}=$ 11.89, $\mathrm{P}<0.01)$.

\subsection{The correlation between the knowledge of nursing students, self-protection behaviors and SAS an- xiety level under theCOVID-19}

Knowledge awareness was positively correlated with self-prevention behavior $(\mathrm{P}<0.05)$, and knowledge awareness was negatively correlated with SAS anxiety $(\mathrm{P}<0.01)$. See Table 3 for details.

Table 3. Correlation between knowledge level of nursing students, self-protection behavior and SAS anxiety level under the COVID-19 $(n=362)$

\begin{tabular}{cccc}
\hline Project & Knowledge level & Self-defense behavior & SAS anxiety level \\
\hline Knowledge level & 1 & - & - \\
Self-defense behavior & $0.126^{*}$ & 1 & - \\
SAS anxiety level & $-0.196^{* *}$ & $-0.202^{* *}$ & 1 \\
\hline
\end{tabular}

Note ${ }^{*} \mathrm{P}<0.05,{ }^{* *} \mathrm{P}<0.01$

\section{Discussion}

\subsection{Analysis of the knowledge of nursing students during the COVID-19}

This study shows that the standardized score of nursing students' knowledge about the COVID-19 is 64.02 points, which is in medium level, and many knowledge points need to be strengthened. Among them, whether ultraviolet rays and chlorine-containing disinfectants can effectively kill the COVID-19 and the COVID-19 period have the lowest scores. The possible reason is that with the development of the epidemic, the clinical features of the disease are constantly being summarized and updated, but some nursing students may not have updated relevant knowledge points in time, and the rendering of false information on the Internet, some nursing students have insufficient professional knowledge and are easily misled by false information. Schools should instruct them to uphold the rigorous and scientific attitude that medical students should have to prevent false perceptions and negative effects caused by false network information at all times. Scholars such as Yang Li [9] through the health education of SARS prevention knowledge for college students, the students' mastery of SARS prevention knowledge has been significantly improved, and their attitudes towards SARS prevention in schools are more positive, indicating that it is helpful to provide relevant knowledge education to college students for epidemic prevention and control during the epidemic. Nursing colleges should provide targeted online education for nursing students on the COVID-19 to increase their knowledge and awareness of major public epidemics. At the same time, the lack of public health education in the training of school nursing talents was exposed during the COVID-19. Relevant education departments should consider adjusting nursing education courses and teaching priorities to improve the ability of nursing students to respond to infection prevention, public health and occupational protection [10].

\subsection{Analysis of self-protection behaviors of nursing students under the COVID-19}

The standardized score of nursing students' self-protection behavior against the COVID-19 is 87.30, indicating that the degree of self-protection of nursing students is relatively high. The survey shows that $90.3 \%$ of students 
will only go out when they have to, $93.7 \%$ of students can wear masks every time they go out, and $64.66 \%$ of students said they would strictly urge their family members to protect themselves, indicating that nursing students can not only do it consciously refusing to go out for activities, wearing masks, maintaining hand hygiene, and health monitoring can also affect family members to actively prevent the epidemic. An investigative study in the United States shows that the protective behavior of college students during the flu period is mainly related to the degree of subjective attention to infectious diseases [11]. The COVID-19 is highly infectious, spreads quickly, and spreads widely. China has adopted prevention and control measures for Class A infectious diseases. The country has actively responded and cooperated. As nursing students with basic medical knowledge, they pay more attention to protection. Besides, the survey found that postponed nursing students lack exercise at home, and only $32.05 \%$ of the nursing students can insist on regular exercise every day. In order to reduce the risk of infection when going out, the increase in indoor time and reduced physical activity will lead to a decrease in immunity.

\subsection{SAS anxiety situation and analysis of nursing students under the COVID-19}

The detection rate of anxiety state of 362 nursing students in this survey was $14.09 \%$, and the SAS anxiety standard score rate of nursing students was $(33.64 \pm 6.17)$ points, which was higher than the Chinese norm $(29.78 \pm$ 10.07) points, and there was obvious anxiety. The reason may be due to the rapid spread of the COVID-19 epidemic and the isolation of nursing students at home, which has led to increasing anxiety of the COVID-19 epidemic. As of April 30, 2020, a total of nearly 3.14 million cases have been confirmed in 212 countries and regions, and a total of about 227,000 deaths have been accumulated. Although the epidemic in China has been basically controlled, people imported from abroad and asymptomatic infections still need to be strictly monitored. Schools should strictly implement prevention and control management following with the law, timely and accurately release the epidemic situation and verify and publish rumors that have caused excessive panic, so as to eliminate students' panic and relieve anxiety; the survey showed that $60.27 \%$ of nursing students said they had experienced the epidemic, had a deeper understanding of nursing, and were more determined to pursue a nursing profession in the future, indicating that under public health emergencies, the students' recognition of the nursing profession is affirmative and firm Dedicated to the career of nursing in the future. However, $2.49 \%$ of the nursing students said that they had a deeper understanding of nursing after experiencing the epidemic, and nursing work itself is risky, so they are less willing to engage in nursing work. For such major public health incidents, the high infection rate, and high-intensity work conditions of medical staff make nursing students who are about to work worried and prone to anxiety. Therefore, relevant nursing schools should actively and positively pass on the deeds of the nursing seniors who participated in the epidemic to the nursing students. In the future, in clinical work, they should correctly apply professional knowledge for scientific protection, achieve standard prevention, and reduce the infection rate. Nursing students are in the learning stage of medical knowledge, their understanding and understanding of diseases are limited to the theoretical level, and they lack practical experience. When faced with public health events, nursing students possessing medical knowledge are more likely to have nervous and fearful emotional reactions [12]. And when young people face an epidemic, their stress response will be stronger [13]. Therefore, schools should increase the proportion of online health education courses, paying particular attention to the mental health education section and the nursing professional commitment section. Quickly evaluate students through the scale, screen out target groups that may have psychological effects in time, and make psychological adjustments to nursing students returning to school to avoid malignant incidents.

\subsection{Correlation analysis of knowledge awareness, self-protection behaviors and SAS anxiety of nursing students under the COVID-19}

Correlation analysis shows that the knowledge awareness of nursing students is positively correlated with self-protection behavior, while the knowledge awareness is negatively correlated with SAS anxiety. This conclusion is consistent with the research results of Li Jing [15]. Under the COVID-19, the more nursing students have knowledge about the COVID-19, the more effective self-prevention behaviors will be adopted, and the anxiety of nursing students due to a major outbreak will also decrease. The self-protection behavior of nursing students has a negative effect on the SAS anxiety level, and can have a negative impact on the anxiety of the nursing students; that is, improving the self-protection behavior can reduce the anxiety level of the nursing students. It can be seen that it is necessary to comprehensively play the role of knowledge awareness and self-protection behavior, publish authoritative new coronavirus-related knowledge, turn knowledge into action, take correct, effective and practical self-protection behavior, and reduce improper protection or inadequate protection The anxiety generated. At present, the ability to prevent and respond to emergencies is being tested. In 2030, global nurses will have a gap of 4.6 mil- 
lion [15]. While investing in the development of nursing education and expanding enrollment, we should also strengthen our ability to respond to emergencies to cultivate. Relevant education departments need to build a combination of online and offline epidemic knowledge system popularization methods, supervise the implementation of self-protection behaviors of nursing students, and provide timely psychological counseling to reduce their anxiety. On the premise of ensuring the authenticity and scientific nature of the epidemic publicity materials, combining the actual situation of the students in this school, the main purpose is to improve the knowledge of the COVID-19 and the compliance of the self-protective behavior of the nursing students, and adopt health education that is more acceptable to the nursing students In the form of training and promotion of the latest authoritative knowledge related to the COVID-19. At the same time, attention should be paid to avoid the concentration of personnel caused by offline preaching, which may cause negative emotions among students [16].

\section{Summary}

The survey found that the self-protection behavior of Chinese nursing students is acceptable, but the level of knowledge about the COVID-19 needs to be strengthened, and some survey subjects show varying degrees of psychological anxiety. It is necessary to build an online and offline combination of epidemic knowledge system popularization methods, supervise and implement self-protection behaviors of nursing students, and screen nursing students with unhealthy emotions, conduct psychological counseling in a timely manner, strengthen professional positive guidance for nursing students, and relieve their unhealthy emotions. Since there is no universal standard questionnaire for COVID-19-related knowledge and protective behavior, the research results are unavoidably affected by the quality of the questionnaire and cannot be compared with other studies. At present, epidemic prevention is becoming normal, and timely understanding of the knowledge of the COVID-19 epidemic of nursing students, and protective behaviors are conducive to the intervention and adjustment of relevant education departments. In subsequent research, the design method can be further improved, the reliability and validity of the scale can be improved, and effective intervention methods can be explored to increase the mental resilience of nursing students to major public health emergencies.

\section{References}

[1] National Health Commission of the People’s Republic of China. (2020). Announcement of the National Health Commission of the People's Republic of China [EB/OL]. (2020-01-20) [2020-03-2020].http://www.nhc.gov.cn/jkj/s7916/202001/ 44a3b8245e80 49d2837a4f27529cd386.shtml.

[2] Choi K R, Heilemann M V, Fauer A, Mead M. (2020). A Second Pandemic: Mental Health Spillover From the Novel Coronavirus (COVID-19) [published online ahead of print, 2020 Apr 27]. J Am Psychiatr Nurses Assoc. 2020;1078390320919803. doi:10.1177/1078390320919803

[3] National Health Commission. (n.d.). The country has sent 42,000 medical personnel to support Wuhan, including 28,600 nurses, accounting for $68 \%$ of the total number of medical teams [EB/OL]. https://mp.weixin.qq.com/s/Bq6x53yfUvVxfgTQCxMfJg.

[4] Ji Xiaoqin, Yu Rong, Mu Min,et al. (2020). Analysis of the psychological status of nursing undergraduates in Sichuan during the novel coronavirus pneumonia epidemic [J]. Medical Education Research and Practice, 2020.

[5] Daly J, Jackson D, Anders R, Davidson P M. (2020). Who speaks for nursing? COVID-19 highlighting gaps in leadership [published online ahead of print, 2020 Apr 20]. J Clin Nurs. 2020;10.1111/jocn.15305. doi:10.1111/jocn.15305.

[6] Chinese Center for Disease Control and Prevention. (2020). Guidelines for public protection against pneumonia caused by novel coronavirus infection [M]. Beijing: People’s Medical Publishing House, 2020.

[7] Klier C M, Geller P A, Ritsher J B. (2002). Affective disorders in the aftermath of miscarriage: A comprehensive review [J]. Archives of Womens Mental Health, 2002, 5(4): 129-149.

[8] Bowles S V, James L C, Solursh D S, et al. (2000). Acute and post-traumatic stress disorder after spontaneous abortion [J]. American family physician, 2000, 61(6): 1689-1696.

[9] Yang Li. (2020). Evaluation of the immediate effect of health education on prevention of SARS for 147 graduates majoring in education [J]. Chinese School Health, 2004(06): 701-702.

[10] Xu Jianmin, Wu Hanquan, et al. (2020). Thoughts on the cultivation of nursing talents from the perspective of the new crown pneumonia epidemic [J]. China Vocational and Technical Education, 2020(11): 57-60.

[11] Van D, McLaws M L, Crimmins J, et al. (2010). University life and pandemic influenza: attitudes and intended behaviour of staff and students towards pandemic (H1N1) 2009. BMC Public Health, 2010, 10: 130.

[12] Zhang Xiaolu, Jia Wei, Duan Liming, et al. (2020). Investigation and analysis of psychological status of 1486 medical students during the period of new coronavirus pneumonia [J/OL]. Journal of Inner Mongolia Medical University. 
[13] Wang Hui, Li Xue, Dun Yue, et al. (2020). Psychological care of adolescents during the epidemic period of new coronary pneumonia [J/OL]. Chinese Mental Health Journal.

[14] Li Jing, Wang Shuhong, Yu Renhe, et al. (2020). Compliance of people's home isolation protection during the new crown pneumonia epidemic and its impact on anxiety [J/OL]. Chinese Journal of Infection Control.

[15] WHO. (2020). State of the world's nursing 2020: Investing in education, jobs and leadership report https://apps.who.int/iris/bitstream/handle/10665/331677/9789240003279-eng.pdf (accessed 18/4/2020).

[16] Zhang Fengying, Li Sheyu, Li Lingli. (2020). Urgent recommendations for university student management in West China during the COVID-19 epidemic [J]. Chinese Journal of Evidence-Based Medicine. 